\title{
Gaps in the interpretation of pronouns*
}

\author{
Keny Chatain \\ MIT
}

\begin{abstract}
Donkey sentences receive either existential or universal truth-conditions. This paper presents two new data points going against standard dynamic approaches to this ambiguity: first, I show that the ambiguity extends beyond quantified environments, to cross-clausal anaphora. Second, I show that donkey sentences can give rise to narrow pseudo-scope readings, where the pronoun's implicit quantification takes scope below some operator in the sentence. Neither of these facts is predicted by standard dynamic accounts. Together, they suggest a different analysis in which the ambiguity arises when the pronoun has multiple referents to pick from. Inspired by Champollion, Bumford \& Henderson (2017), I propose that when such circumstances arise, the pronoun receives vague reference. Using standard rules of projection is then sufficient to derive the existential/universal ambiguity as well as the two problematic data points.
\end{abstract}

Keywords: donkey sentences, strong/weak readings, vagueness, cross-conjunction anaphora

\section{Introduction}

Three problems. The sentences in (1-2) raise a number of problems for theories of pronoun interpretation. The first problem is to explain how an indefinite and a pronoun may co-vary, when the latter is not in the scope of the former. Two particular cases are often discussed: donkey sentences (Geach 1964), such as (1), where the indefinite and the pronoun are split between the restrictor and the nuclear scope of a quantifier, and cross-clausal anaphora (2) where the indefinite and the pronoun are in different but conjoined clauses.

(1) a. Every farmer who owns a donkey treats it gently.

b. No student who wrote a thesis on algebra was satisfied with it.

c. Some adventurer who found a dinosaur bone donated it to the museum.

a. Camelia has a donkey and she cherishes it.

* For valuable feedback and discussion, I thank Gennaro Chierchia, Lucas Champollion, Michel DeGraff, Edward Flemming, Danny Fox, Martin Hackl, Irene Heim, Roger Schwarzschild, and the students of the MIT Workshop of Fall 2017 and anonymous SALT reviewers. Special thanks to Lucas Champollion for extensive discussion of both form and content.

CC2018 Chatain 
b. Camelia has a donkey and she cherishes it.

c. If Camelia adopts a donkey and she cherishes it, she will feel bliss.

The second problem raised by such sentences is determining what truth conditions they receive. For instance, it is known that sentences containing every may receive two different sets of truth conditions: the universal reading and the existential reading. This is shown in (3) and (4).

(3) Every farmer who owns a donkey treats it gently.

UNIVERSAL $\leftrightarrow$ every donkey-owning farmer treats all of his donkeys gently.

(4) Every person who had a hat wore it at the party.

EXISTENTIAL $\leadsto$ every hat-owning person wore one of his hats.

The final challenge raised by these sentences is to understand what pragmatic factors affect the availability of the different readings. Some generalizations seem to hold (see Kanazawa 1994 for discussion, and Foppolo 2008 for experimental evidence): for instance, it seems that quantifiers come with a preferred reading: universal for "every", existential for "some" and "no".

Goal. In this paper, I intend to tackle the second challenge, namely that of figuring what the underlying truth-conditions of the sentences in (1) and (2) are. Particularly relevant to this paper is the existence and form of the existential/universal ambiguity. I will first make a negative contribution, by providing evidence that accounts that rely on the interpretation of dynamic conjunction to obtain the existential/universal ambiguity discussed above face two under-generation issues: first, they fail to predict cases of narrow pseudo-scope readings (as I will refer to them), where the quantificational force associated with the pronoun seems to be able to take scope beneath other operators in the sentence; second, I will show that the existential/universal ambiguity in fact extends beyond donkey sentences, and can be replicated with cross-clausal anaphoras as in (2), despite initial evidence to the contrary and previous literature. If these arguments are successful, they put in question the usefulness of common dynamic denotations for conjunction.

Taken together, these two under-generation issues seem to point in the same direction: they suggest that the existential/universal ambiguity originates from the pronoun. I will accept this fact and propose the following explanation to it: pronouns that have multiple referential targets, as do pronouns anteceded by indefinites with more than one witness, have vague reference. The treatment of vagueness is the trivalent semantics offered in Champollion et al. 2017, supplemented with a projection rule. The resulting theory will correctly predict existential/universal ambiguity across the board and narrow pseudo-scope readings. 
Gaps in the interpretation of pronouns

This theory comes with two caveats: first, it needs to be supplemented with a theory of vagueness resolution. The procedure in Champollion et al. 2017 is readily available, but future research will be needed to explain the existence of the preferred readings discussed before (the third challenge). Second, for simplicity, I will make commitments as to how a pronoun access its referents and adopt an E-type strategy. As I will explain, this does not mean that a dynamic version of the theory is out of question.

Outline. In section 2, I will introduce what I will call the Extension Principle, a principle enforced by common rules of interpretation for conjunction in Dynamic Semantics, and show how it is often used to craft quantifiers that derive the existential and universal readings. In section 3, I will present two under-generation problems, and show how they derive from the adoption of the extension principle. I will then proceed to present my own analysis in section 4 . I will then discuss previous approaches to the phenomenon in section 5 and conclude.

\section{Extension principle: the interpretation of dynamic conjunctions}

Extension principle. Standard dynamic systems are designed to give conjoined clauses like (5a) the same truth-conditions as a sentence in which the indefinite takes scope over the conjunction, such as (5b). This principle, stated below, is what I will refer to as the Extension Principle ${ }^{1}$.

a. Camelia has a donkey and she cherishes it.

b. There is a donkey that Camelia has and that she cherishes.

$$
\begin{aligned}
& \text { Extension Principle } \\
& (\exists x, A(x)) \wedge B(x) \Leftrightarrow \exists x,(A(x) \wedge B(x))
\end{aligned}
$$

This principle is not basic; it is often derived from two more primitive ingredients: non-deterministic updates performed by the indefinite and an adequate rule of interpretation for conjunction that channels these updates. In the sequel, it will not be necessary for us to know how those ingredients work in detail ${ }^{2}$. I will simply take it for granted that the denotation of conjunction and the indefinite work together to give rise to this principle.

1 This principle is stated at the meta-language level. This is suitable for dynamic systems which translate natural language statement into logics such as Dynamic Predicate Logic (Groenendijk \& Stokhof 1991). In dynamic systems where the interpretation function maps the object language directly onto model-theoretic objects, such as Compositional DRT (Muskens 1996), the Extension Principle would need a harmless restatement in terms of those model-theoretic objects.

2 See Groenendijk \& Stokhof 1991 and Muskens 1996 for a presentation. 
Consequences. By design, as we've seen, such a principle predicts the possibility of cross-conjunction anaphora and furthermore predicts that cross-conjunction anaphora should get existential readings. But the principle has consequences beyond cross-conjunction anaphora: readings of donkey sentences of the likes of (6) can naturally be constructed on the basis of the Extension Principle.

(6) Every farmer who owns a donkey treats it gently.

To do so, one needs to capitalize on the logical equivalence in (7), which holds for conservative quantifiers. This makes it possible to create the truth-conditionally equivalent denotation for natural language quantifiers in (8), where the scope of the quantifier now encompasses both the NP content and the VP content. In the case of donkey sentences, this results in a conjunction of a clause with an indefinite and a clause containing a pronoun. By the Extension Principle, we know that the pronoun in the resulting structure will be interpretable and will be interpreted existentially (cf (8b), derivation in (8)). In short, this denotation makes existential donkey anaphora possible.

(7) If $Q$ is a conservative quantifier, $Q$ (restrictor)(scope) is equivalent to $Q($ restrictor $)($ restrictor $\wedge$ scope $)$

$\llbracket$ every $\alpha \beta \rrbracket=\forall x \in \llbracket \alpha \rrbracket, \llbracket \alpha \rrbracket(x) \wedge \llbracket \beta \rrbracket(x)$

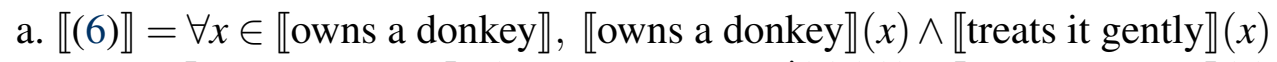
$=\forall x \in \llbracket$ owns a donkey $\rrbracket,(\exists y$, owns-donkey $(y)(x)) \wedge \llbracket$ treats it gently $\rrbracket(x)$ $=\forall x \in \llbracket$ owns a donkey $\rrbracket, \exists y,\left(\right.$ owns-donkey $(y)(x) \wedge$ treats-gently $\left.^{\prime}(y)(x)\right)$ (applying the Extension Principle)

b. $(6) \rrbracket=$ true iff for every donkey-owning farmer treats gently some donkey he owns

This is not the only way to create a donkey-friendly version of quantifiers. The logical equivalence in (9) provides another way. Again, thanks to the Extension Principle, it is possible to see that this sentence will be interpretable and that it yields a universal reading of the pronoun.

(9) If $Q$ is a conservative quantifier, $Q$ (restrictor)(scope) is equivalent to $Q($ restrictor $)(\neg($ restrictor $\wedge \neg$ scope $))$

$\llbracket$ every $\alpha \beta \rrbracket=\forall x \in \llbracket \alpha \rrbracket, \neg(\llbracket \alpha \rrbracket(x) \wedge \neg \llbracket \beta \rrbracket(x))$

a. $\llbracket(6) \rrbracket=$ true iff for every donkey-owning farmer treats gently every donkey he owns

The conclusion to draw from this discussion is that in dynamic semantics, the analysis of quantified sentences - and thus donkey sentences - follows smoothly from the 
Gaps in the interpretation of pronouns

treatment of cross-conjunction anaphora. Moreover, the two recipes for deriving dynamic quantifiers coincide with the desired ambiguity between existential/universal readings ${ }^{3}$.

There are however good reasons why such an account of the ambiguity has been abandoned in subsequent dynamic literature. One such reason is the availability of mixed readings (Brasoveanu 2007), as in (11), where a single quantifier hosts in its scope both an existentially interpreted pronoun and a universally interpreted pronoun. Any account that derives the existential/universal ambiguity from a similar ambiguity in the quantifier's denotation would fail to make that prediction.

(11) Every gentleman who has a credit card 1 and bought a yacht t $_{2}$ used the card ${ }_{1}$ to pay for the yacht 2 .

$\rightsquigarrow$ used $a$ card of his for every yacht he bought.

Be it as it may, alternative dynamic accounts of the ambiguity, which we will review in section 5, make use of at least one of the two dynamic quantifier denotations that we discussed, along with other assumptions. As such, they fall victim to the challenges I now proceed to present.

\section{Challenges to the extension principle}

Universal readings across clauses. As we saw, the Extension Principle only generates existential readings for cross-clausal anaphora. This is in line with previous literature, which only acknowledges the existence of existential readings (as in (12)) to cross-clausal anaphora. My goal is to show that universal readings also exist.

(12) Camelia has a donkey and she cherishes it.

$\rightsquigarrow$ Camelia has a donkey and she cherishes one of the donkeys she owns.

Before that, let me explain why we a priori expect that such a reading would be difficult to find. Recall from the introduction that donkey sentences constructed with quantifiers no and some seem to prefer an existential reading. This is only a preference, however; it has been noted (Kanazawa 1994; Chierchia 1995; Geurts 2002) that certain biasing items - typically those containing some flavour of negation ${ }^{4}$ - can produce universal truth-conditions, as revealed by the example below.

(13) No gentleman who owns an umbrella left it at home today.

$\rightsquigarrow$ no umbrella-owning gentleman left all of his umbrellas

3 These two recipes for constructing dynamic quantifiers have been known since at least Rooth 1985 , Root 1986.

4 The verb leave can be construed as the negation of "take" and thus lumped with negative elements. This step would require a justification; it is not immediate on theoretical grounds that leave should be the negative pole of the antonym pair take-leave. 
The conclusion to draw from this discussion of donkey sentences is the following: whenever a donkey sentence has a preferred existential reading, the universal reading is marginal and is only revealed by biasing items. A natural expectation to have is the following: if the existential/universal ambiguity does exist in cross-clausal contexts, it is hard to find and requires careful choice of lexical items.

There is another hurdle in the way of discovering universal readings: uniqueness implicatures. A unembedded indefinite as in (12) frequently give rise to a uniqueness implicature (e.g. Camelia owns just one donkey), thus collapsing the difference between existential and universal readings. In the following tests, I will therefore use bets (Schlenker 2012) to probe unstengrenthened truth-conditions. (14) shows that this technique is sufficient to remove uniqueness implicatures.

(14) a. I bet you $\$ 10$ that Mrs. Jones will buy a car

b. Outcome: Mrs. Jones bought two cars. $\rightsquigarrow$ speaker wins

Now consider the bets in (15-16), with biasing items and appropriate contextualizing. Under the existential reading that the Extension Principle predicts, they should both be won under the described outcomes, since one can find an umbrella that Camelia has and that she left, or a black suit that Charles packed and that he is not wearing.

a. Context: Speaker is arguing that Camelia is absent-minded, hearer disagrees.

b. I bet you $\$ 10$ that Camelia has an umbrella and that she left it at home today.

c. Outcome: Camelia has 10 umbrellas. She brought one and left the others.

a. Context: Speaker suspects Charles is not following the boat party's dresscode, hearer disagrees.

b. I bet you $\$ 10$ that Charles packed a black suit and that he is not wearing it right now.

c. Outcome: Charles packed two black suits and was wearing one of them at the time of the bet.

The reactions I collected for these bets were not unanimous but more importantly, they were all inconsistent with what the Extension Principle predicts. One population of speakers thought the bet was categorically lost. Another population agreed with the loss verdict but pointed out that a combative speaker may want to point out that the umbrellas that were left or the unworn suits. One speaker thought the bet was off, for lack of a clear outcome. Finally, only one speaker thought the bet was won. 
Gaps in the interpretation of pronouns

To appreciate this variation, it is useful to compare these reactions to the baselines obtained by scoping the indefinite above the conjunctions. Here, informants unanimously grant victory to the speaker. Under the Extension Principle, the two sets of bets should have the same truth-conditions and this discrepancy is unexpected.

a. I bet you $\$ 10$ that there is an umbrella that Camelia has and that she left at home.

b. Outcome: Camelia has 10 umbrellas. She brought one and left the others. $\rightarrow$ won!

a. I bet you $\$ 10$ that there is a black suit that Charles packed and that he is not wearing right now.

b. Outcome: Charles packed two black suits and was wearing one of them at the time of the bet. $\rightarrow$ won!

In conclusion, we see that when using biasing items, a majority of speakers seem to be able to access stronger universal truth-conditions. Under the account to be presented, both this judgment and the variation observed in the range of responses will find an explanation. Indeed, I will propose that cross-clausal anaphora are intrinsically vague. This means that the bets studied in (15-16) are vague. Since different speakers may have different strategies to cope with this vagueness, the responses are expected to be speaker-dependent.

Narrow pseudo-scope readings. All the paraphrases for the existential/universal ambiguity I gave so far replace the pronoun by some quantified expression, either existential or universal. Let's refer to this quantifier as the implicit quantifier. Its scope will be called the pseudo-scope of the pronoun, following Brasoveanu $2007^{5}$.

An interesting property of the Extension Principle is that it only generates wide pseudo-scope readings. To see that, imagine a sentence like (19a), where OP is some operator capable of displaying scope interactions. By the Extension Principle, this sentence is to be interpreted as in (19b). In this reading, the pronoun's implicit quantification is existential and has pseudo-scope over OP. This is the only possibility.

$$
\begin{aligned}
& \text { a. }\left(\ldots[\mathrm{a} \mathrm{NP}]_{\mathrm{i}} \ldots\right) \text { and }\left(\ldots \mathrm{OP} \ldots \mathrm{it}_{\mathrm{i}} \ldots\right) \\
& \text { b. } \exists x \in \llbracket \mathrm{NP} \rrbracket,\left(\ldots x_{\mathrm{i}} \ldots\right) \text { and }(\ldots \mathrm{OP} \ldots x \ldots)
\end{aligned}
$$

In light of the discussion of section 2, we know that this result carries over to donkey sentences, since the readings of the latter are constructed by the Extension Principle. So (20) will be interpreted as either (20a) or (20b).

5 These labels are only meant as useful terms to describe readings; as such, they do not come with any theoretical assumptions about the interpretation of pronouns. 
(20) Every farmer [that owns a donkey] [... OP ... it $\left.\mathrm{i}_{\mathrm{i}} \ldots\right]$

a. Every farmer [that owns a donkey] $[\forall x$, (farmer owns $x) \rightarrow \ldots$ Op . . . . . ] ]

b. Every farmer [that owns a donkey] $[\exists x$, (farmer owns $x$ ) $\wedge \ldots$ Op ... $\ldots$. ]

To recap, the Extension Principle predicts implicit quantification of the pronoun to have the widest pseudo-scope in cross-clausal anaphora, as well as in donkey sentences. Does this prediction hold? No, as it turns out. To my knowledge, Solomon (2012) was the first to make the observation ${ }^{6,7}$. He considers the sentence in (21), which contains the scope-bearing operator forget. He notices that the truth-conditions of (21) are not quite those given by the wide-pseudo-scope paraphrase in (21a), since these entail the existence of a specific quarter that each man was supposed to put in the meter. Rather, it seems that the truth-conditions simply entail that each man was supposed to put some quarter or other in the meter (cf (21b)). This is a narrow pseudo-scope reading.

(21) At least one man who had a quarter forgot to put it in the meter.

a. At least one man who had a quarter is such that there is some quarter he has that he forgot to put in the meter.

b. At least one man who had a quarter is such that he forgot to put some quarter or other in the meter.

Other examples can be constructed with more mundane scope-bearing operators. In each case, the paraphrase features an implicit quantification taking scope below the boldfaced operator. For instance, (22) can be true even if a particular movie aficionado uses different passes on different occasions and (23) when some father used different chocolate bars for different children.

6 I thank Simon Charlow for this reference.

7 Solomon's (2012)'s own solution to the problem is dynamic in nature. He proposes that the referent's introduction property of an indefinite may be delayed, up till the point of evaluation of the pronoun. When such a delay is created, the pronoun's implicit quantification has scope as low as the pronoun and is necessarily existential. When no delay is created, the pronoun behaves according to the rules set in section 2. In this system, the choice of delaying has to be made at the level of the indefinite, since it is the indefinite which introduces the referents. As such, it will fail to predict mixed cases where a single indefinite antecedes both a wide-pseudo-scope pronoun interpreted with universal force and a low-pseudo-scope pronoun with universal force.

(i) Every citizen who has an ID keeps it in his wallet and shows it whenever she is asked to.

$\rightsquigarrow$ keeps all of his IDs in his wallet.

$\rightsquigarrow$ shows one of his IDs when she is asked.

Similar kind of counter-evidence will be discussed when discussing Brasoveanu 2008 in section 5. 
Gaps in the interpretation of pronouns

(22) Every citizen who has a movie pass ${ }_{1}$ uses it ${ }_{1}$ every chance he has.

$\rightsquigarrow$ for every chance he has, he uses one of the movie passes he has.

(23) Every man who received a chocolate bar ${ }_{1}$ gave a piece of it 1 to each of his kids

$\rightsquigarrow$ for every kid, he gave a piece of one of the chocolate bars he received.

The narrow pseudo-scope reading also obtains in the cross-clausal case, as is expected from section 2; one just needs to make sure to cancel the uniqueness implicature by using a bet environment.

(24) I bet you $\$ 10$ that Charles has a valid ID and that he shows it whenever he is asked to.

(25) Outcome: Charles has two valid ID cards and shows one or the other whenever he is asked. $\rightsquigarrow$ won!

Recap. In this section, I have shown that two predictions made by the Extension Principle were not borne out. The first prediction was the absence of universal readings for cross-clausal anaphora. The second prediction was the obligatory wide pseudo-scope of the implicit quantifier. The immediate conclusion to draw is that there's something in the truth-conditions of anaphora anteceded by indefinites which is not captured by standard dynamic systems.

Taking a step back, one can see another conclusion emerging from the discussion. The presence of universal readings in cross-clausal anaphora suggests that the existential/universal ambiguity exist across the board (as soon as the pronoun is anteceded by an indefinite). It is thus independent of the donkey environment. As for the existence of narrow pseudo-scope readings, it suggests that the ambiguity is, in some sense, local to the pronoun. Whatever is responsible for creating the implicit quantification can see the environment the pronoun is in. Putting these two conclusions together suggests a different take on the truth-conditions of anaphora anteceded by an indefinite and the existential/universal ambiguity: it is the pronoun itself that is responsible for the ambiguity. In the next section, I'll present an analysis exploring that particular route. Specifically, I will claim that when there are multiple referents that a pronoun can refer to, the pronoun has a vague interpretation. It is this vagueness that is to blame for the ambiguity. Since the vagueness is generated locally to the pronoun, it is able to generate narrow pseudo-scope readings.

\section{Analysis}

The idea that we want to capitalize on is that the ambiguity arises when speakers are unable to select a unique referent for a particular pronoun; in such a situation, 
vagueness ensues, of a trivalent nature, following ideas from Champollion et al. 2017. As there are different ways this vagueness may be resolved in context, the sentence consequently receives different readings, accounting for both the ambiguity and the examples discussed in the previous section.

\subsection{Basic E-type set-up}

Before I am able to spell out this intuition, I will first need to take a stand on how a pronoun accesses its antecedent. Here for simplicity, I will adopt the E-type approach of Cooper 1979. At the end of the section, I will explain how much commitment there really is to this choice, for anyone who embraces the vagueness approach advocated here. In the approach of Cooper 1979, pronouns contain a covert structure of the form in (26). In this structure, the index $i$ denotes the individual $g(i)$, that is provided by the assignment function, $R$ is a contextually salient relation, and the is the definite article.

$$
\mathrm{it}_{\mathrm{i}}=[\text { the }[\mathrm{R} \mathrm{i}]]
$$

In standard donkey sentences, the E-type approach assumes that $i$ is bound by the quantifier "every farmer" and that $\mathrm{R}$ is resolved to the relation described in (27).

(27) Every farmer who owns a donkey treats $\mathrm{it}_{\mathrm{i}}(=\mathrm{the} \mathrm{R} \mathrm{i})$ well. $\rightsquigarrow R(x)(y)$ iff $y$ is a donkey owned by $x$

(28) Reading: every donkey-owning farmer treats the donkey that he owns well

There is a known problem to this approach (Heim 1982): under a standard Fregean analysis of the, the sentence should carry a uniqueness presupposition that every farmer owns only one donkey. The fact that such uniqueness fails to hold in donkey sentences is precisely why we come to be interested in the existential/universal ambiguity in the first place.

In short, the problem with Cooper's (1979)'s approach is that it imposes a strong condition that we may uniquely identify the donkey that each farmer owns. The general philosophy of my approach, stated in the introduction, is different in spirit to the Fregean analysis: when donkeys may not be uniquely identified, vagueness ensues, not infelicity. To clearly differentiate the vague definite article I propose from the Fregean uniqueness definite article, I will refer to the former as $d$ the.

\subsection{Vagueness}

Vague pronouns. When a sentence like (29a) is evaluated in a world where Joey own three donkeys Twix, Crunch, and Mars, we want to say that the hearer will be 
Gaps in the interpretation of pronouns

unsure whether it refers to Twix, Crunch or Mars. Formally, this will be implemented by having it denote the set of all three individuals as in Alternative Semantics ${ }^{8}$ (cf (29b)) .

(29) a. Joey i owns a donkey and he treats it (=dthe $R i$ ) well

b. $\llbracket \mathrm{it}_{\mathrm{i}} \rrbracket=\left\{\mathrm{twix}^{\prime}, \operatorname{crunch}^{\prime}, \operatorname{mars}^{\prime}\right\}$

How is this result derived from the basic structure of the E-type pronoun? I submit that $d$ the is itself the vague item in the structure of the pronoun. In the pronoun structure, the constituent [ $\mathrm{R}$ i] represents a set of possible referents. The item $d$ the represents a way of choosing a referent. Since there are many ways of choosing a referent in a set, $d$ the will have multiple outputs, yielding the desired vagueness.

This mechanism of choice is implemented using choice functions (Reinhart 1997); a choice function is a function from set of individuals to individuals in that set. I assume that dthe denotes all such choice functions. This is the inherent vagueness of $d$ the.

$$
\llbracket \text { dthe } \rrbracket=\{f \mid \forall E \neq \emptyset, f(E) \in E\}
$$

The combination of $d t h e$ with the constituent [R $\mathrm{i}]$ is obtained with the rule of pointwise functional application rule, given below. This rule is the standard replacement of functional application in the context of Alternative Semantics.

Functional Application. If $A$ is a node of type $b c$ and $B$ a node of type $b$, then:

$$
\llbracket A B \rrbracket^{g, w}=\left\{f(x) \mid f \in \llbracket A \rrbracket^{g, w}, x \in \llbracket B \rrbracket^{g, w}\right\}
$$

Trivalent truth-conditions. Within this alternative semantics, the second conjunct of (29a) receives multiple truth-values, depending on which referent for $i t_{i}$ is chosen to pursue the evaluation. A derivation of the second clause in (29a) is provided in (31) assuming that Twix, Crunch and Mars are Joey's donkeys. The net result of it, given in (32), is the following: in a world where Joey treats Twix well, but not the other donkeys, the sentence receives two truth-values: $\{$ true, false $\}$. By convention, we denote this set as \#. In a world where he treats all of his donkeys well, the sentence only receives the truth-value true; finally the sentence only receives the truth-value false in a world where Joey treats all of his donkeys poorly.

a. $\llbracket$ treats it well $\rrbracket^{g, w}=$ $\{\lambda x . x$ treats Twix well in $w, \lambda x . x$ treats Crunch well in $w$, $\lambda x . x$ treats Mars well in $w\}$

8 See Kamp \& Partee 1995 for another use of alternative semantics to deal with vagueness. 
b. $\llbracket$ treats it well $\rrbracket^{g, w}=$

$\{$ Joey treats Twix well in $w$, Joey treats Crunch well in $w$, Joey treats Mars well in $w$ \}

$\llbracket$ he treats it well $\rrbracket^{g, w}=\left\{\begin{array}{cl}\text { true } & \text { if Joey treats all of his donkeys well. } \\ \text { false } & \text { if Joey treats none of his donkeys well. } \\ \# & \text { if Joey treats some but not all of his donkeys well. }\end{array}\right.$

This is the desired result: failure of uniqueness does not trigger infelicity as in Cooper 1979 but vagueness. This vagueness may or may not result in uncertainty about truth-values, depending on which world the sentence is evaluated in.

Resolving vagueness. Now, we will see how the truth/falsity-conditions above translate into an existential/universal ambiguity. When the speaker expresses a proposition which evaluates to \# in certain worlds from the common ground $^{9}$, hearers need to decide whether these \#-worlds are to be included in the new updated context set or excluded from it. To put it differently, hearers have to give \#-world to an actual truth-value. This decision is pragmatic in nature and reflects hearer's expectations of what the speaker intended to express.

Whatever the pragmatic reasoning is that hearers follow, existential truthconditions are obtained when hearers uniformly assign true to \#-worlds. Indeed, from (32), this amounts to saying that the sentence is true just in case Joey treats some but not all or all of his donkeys well; in other words, the negation of the falsity conditions. Similarly, universal truth-conditions result from uniformly assigning false to \#-worlds. In a nutshell, cross-conjunction anaphora can get both existential and universal readings.

Of course, a complete account needs to specify exactly what procedure hearers follow and what conversational situations lead to one reading or the other. This ties back to the problem mentioned in the introduction of deriving the default readings of donkey sentences. Champollion et al. (2017), using ideas from Križ 2016, provide such a procedure. Their procedure relies on the question under discussion to recover the missing truth-values. In default contexts, the question under discussion is assumed to be maximally fine-grained and the resulting reading the strongest reading possible. To account for some, whose default reading is existential, and therefore the weakest reading possible, they adopt the proposal by Geurts (2002) that intersective quantifiers can be evaluated against sub-models, effectively turning universal readings into existential ones.

I have nothing to add to this proposal, which can be adapted wholesale to the current system. However, since my goal is to design a system that generates all

9 I assume the reader is familiar with the Stalnakerian model of conversation, where propositions are used to update the common ground, i.e. the set of shared beliefs. 
Gaps in the interpretation of pronouns

observed interpretations of donkey sentences and not to derive reading preferences, I will set the gap resolution procedure aside in the rest of this article.

Donkey sentences. To deal with donkey sentences and make the pseudo-scope predictions, it is necessary to specify how vagueness interacts with $\lambda$-abstraction. Fortunately, there is a ready-made rule in the alternative semantics literature rule that happens to fit our needs. It is defined in Kratzer \& Shimoyama 2017. The rule states that one forms a set of predicate denotations from a set of proposition denotations containing an unbound variable $x$, by taking all the different ways of mapping $x$ to an element of the alternative proposition set.

$\lambda$-abstraction rule. If $A$ is a constituent of type $t$, then $\llbracket \lambda_{i} A \rrbracket^{g, w}=\left\{f \in D_{e t} \mid \forall x, f(x) \in \llbracket A \rrbracket^{g, w}\right\}$

As an example, we'll derive the truth-falsity conditions of the simple donkey sentence in (33). Let's assume that the context provides the value " $\lambda x . \lambda y . y$ is a donkey owned by $x$ " for $R$. The derivation is given in (33).

(33) Every farmer who owns a donkey $\lambda_{1} t_{1}$ treats it(=dthe $R i$ ) well.

(34) a. The interpretation of the pronoun is the set of all donkeys owned by a given farmer

$$
\begin{aligned}
\llbracket \text { the } \mathrm{R} 1 \rrbracket^{g[1 \rightarrow x]} & =\llbracket \text { the } \rrbracket\left(\llbracket \mathrm{R} 1 \rrbracket^{g[1 \rightarrow x]}\right) \\
& =\left\{y \text { is a donkey owned by } x \mid y \in D_{e}\right\}=: \operatorname{Donk}(x)
\end{aligned}
$$

b. The nuclear scope receives the same trivalent truth-conditions as in the cross-conjunction example.

$$
\begin{aligned}
\llbracket t_{1} \text { treats it well } \rrbracket^{g[1 \rightarrow x]} & =\llbracket \text { treats-well } \rrbracket^{g[1 \rightarrow x]}\left(\llbracket \text { it } \rrbracket^{g[1 \rightarrow x]}\right)\left(\llbracket t_{1} \rrbracket^{g[1 \rightarrow x]}\right) \\
& =\{x \text { treats } y \text { well } \mid y \in \operatorname{Donk}(x)\} \\
& = \begin{cases}\text { true } & \text { if for all } y \text { in } \operatorname{Donk}(x), x \text { treats } y \text { well } \\
\text { false } & \text { if for no } y \text { in } \operatorname{Donk}(x), x \text { treats } y \text { well } \\
\# & \text { otherwise }\end{cases}
\end{aligned}
$$

c. Abstracting over index 1 following the abstraction rule given above.

$$
\begin{aligned}
\llbracket \lambda_{1} t_{1} \text { treats it well } \rrbracket^{g} & =\left\{f \in D_{e t} \mid \forall x, f(x) \in \llbracket t_{1} \text { treats it well } \rrbracket^{g[1 \rightarrow x]}\right\} \\
& =\left\{\begin{array}{ll}
f \mid \begin{array}{l}
f(x)=\text { true if } x \text { treats well all } y \text { in } \operatorname{Donk}(x) \\
\text { and } f(x)=\text { false if } x \text { treats well no } y \text { in Donk }(x)
\end{array}
\end{array}\right\}
\end{aligned}
$$


d. Finally, the quantifier combines with this alternative predicate:

$$
\begin{aligned}
\llbracket(33) \rrbracket^{g} & =\llbracket \text { every farmer } \ldots \rrbracket\left(\llbracket \lambda_{1} t_{1} \text { treats it well } \rrbracket^{g}\right) \\
& =\left\{f \in D_{e t} \mid \forall x, f(x) \in \llbracket t_{1} \text { treats it well } \rrbracket^{g[1 \rightarrow x]}\right\} \\
& = \begin{cases}\text { true } & \text { if every farmer } x \text { who }[. . .] \text { treats well every } y \text { in } \operatorname{Donk}(x) \\
\text { false } & \text { if some farmer } x \text { who }[. . .] \text { treats well no } y \text { in } \operatorname{Donk}(x) \\
\# & \text { otherwise }\end{cases}
\end{aligned}
$$

Just as with the cross-conjunction case, the existential and universal reading correspond to two different assignment of truth-values to \#-worlds. When all the \#-worlds are assigned to false, the only worlds that are true are those that were initially true; by (34d), this corresponds to the reading "every farmer $x$ who owns a donkey treats well every $y$ in $\operatorname{Donk}(x)$ ". This is the universal reading. When all the \#-worlds are assigned to false, all the worlds that were initially not false (i.e. true or \#) are treated as true. The resulting reading is the negation of the falsity conditions: "not some farmer $x$ who owns a donkey treats well no $y$ in $\operatorname{Donk}(x)$ ", i.e. "every farmer $x$ who owns a donkey treats well some y in $\operatorname{Donk}(x)$ ". In other words, the existential reading.

Narrow pseudo-scope. Our predictions diverge from that of classical dynamic semantics in exactly the problematic cases. Since the vagueness stems from the pronoun, any operator that the pronoun is in the scope of may affect the truth-falsity condition, yielding the observed narrow pseudo-scope. Consider (35). I derive the predicted truth-conditions, focusing on the important steps.

(35) Every citizen who has a valid ID $\lambda_{1} t_{1}$ shows it (=dthe R 1 ) whenever she ${ }_{1}$ is asked to.

(36) a. Assume that whenever is a universal quantifier over a time domain $A$

$$
\llbracket \text { whenever she }{ }_{1} \text { is asked to } \rrbracket^{g, \tau_{0}}=\lambda_{\tau t} . \forall t \in A, p(t)
$$

b. Following the same steps as above:

$$
\llbracket t_{1} \text { shows it } \rrbracket^{g[1 \rightarrow x], t}= \begin{cases}\text { true } & \text { if } x \text { shows all } y \text { in } \mathbf{I D}(x) \text { at } t \\ \text { false } & \text { if } x \text { shows no } y \text { in } \mathbf{I D}(x) \text { at } t \\ \# & \text { otherwise }\end{cases}
$$

c. The vagueness projects out of the whenever quantification similar to (34d)

$$
\begin{aligned}
& \llbracket t_{1} \text { shows it whenever ... } \rrbracket^{g[1 \rightarrow x], \tau_{0}}= \\
& \begin{cases}\text { true } & \text { if for all } t \text { in A, } x \text { beat all } y \text { in } \mathbf{I D}(x) \text { at } t \\
\text { false } & \text { if for some } t \text { in } \mathrm{A}, x \text { beat no } y \text { in } \mathbf{I D}(x) \text { at } t \\
\# & \text { otherwise }\end{cases}
\end{aligned}
$$


Gaps in the interpretation of pronouns

d. Likewise, we derive the matrix level truth/falsity conditions.

$$
\llbracket(35) \rrbracket^{g[1 \rightarrow x], \tau_{0}}= \begin{cases}\text { true } & \begin{array}{l}
\text { if for all ID-owning citizen } x, \text { for all } t \text { in A, } \\
\text { for all } y \text { in ID }(x), x \text { shows } y \text { at } t
\end{array} \\
\text { false } & \begin{array}{l}
\text { if for some ID-owning citizen } x, \text { for some } t \text { in A, } \\
\text { for no } y \text { in ID }(x), x \text { shows } y \text { at } t \\
\text { otherwise }
\end{array}\end{cases}
$$

Let us unpack the predictions. As with donkey sentences, existential truth-conditions are obtained when all the \#s are resolved to false. So the truth-conditions of the existential reading can be obtained by simply negating the falsity conditions of the sentence. This gives the narrow pseudo-scope existential reading in (37).

(37) (35) is true under the existential reading iff every ID-owning citizen showed some ID or other every time she was asked

\subsection{Recap}

In this section, we spelled out the idea that pronouns are responsible for the existential/universal ambiguity. We assumed that when a pronoun's antecedent spans multiple referents, the pronoun is itself ambiguous between these referents. We gave two standard rules of Alternative Semantics governing the way vagueness of reference projects at the level of the sentence. The resulting truth/falsity conditions were shown to result in existential/universal ambiguity for simple donkey sentences. The analysis went further than that and derived existential/universal ambiguity for cross-conjunction anaphora and narrow pseudo-scope readings, the two problematic cases of section 3 .

What is crucial to this approach is the vagueness and the rules of projection thereof. As for the E-type component, its contribution can be summed up as follows: it provides the pool of referents (e.g. the donkeys owned by Camelia) which dthe can then choose from. One could therefore imagine to replace the E-type part of the analysis by a dynamic system where the set of all donkeys owned by Camelia would all be stored in an index ${ }^{10}$. In this system, the pronoun would choose a referent from this index in much the same way as $d$ the chose from the individuals provided by the descriptive content of the pronoun. If such a system were to be adopted, the predictions would be identical and the intuitions behind the system would be preserved.

10 This is reminiscent of the system in Solomon 2012 (see fn. 7). Two majour differences are that the choice of the pronoun's implicit quantification need not be made at the level of the indefinite and the vagueness mechanics. 


\section{Previous approaches}

In this section, I review two approaches to the existential/universal amiguity and compare them to my own: Champollion et al. 2017, the inspiration of this work, and Brasoveanu 2008, which also discusses narrow pseudo-scope readings.

PCDRT. In a series of works (Brasoveanu 2007, 2008; Brasoveanu \& Farkas 2011), Brasoveanu develops the Plural Compositional DRT framework, which relies on the plural assignments conceived by van den Berg 1996. He shows that this innovation can be used to account for an impressive range of discourse phenomena. Of particular interest to us is his treatment of the existential/universal ambiguity. His proposal is to distinguish between a weak and a strong indefinite; the former is responsible for existential readings and the latter for universal readings. As noted by Champollion et al. (2017), this predicts that a single indefinite may not antecede both an existentially interpreted pronoun and a universally interpreted pronoun. This is not borne out as the example from Champollion et al. 2017, repeated in (38), shows.

(38) Every man who has an umbrella takes it along on rainy days but leaves it home on sunny days.

$\rightsquigarrow$ takes one of his umbrellas ... leaves all of his umbrellas

In addition to this, the present work provides different challenges to the system in Brasoveanu 2008. In spite of the adoption of plural assignments, the system cannot generate universal cross-conjunction readings and narrow pseudo-scope readings. Regarding the former, PCDRT allows for two structures depending on whether the indefinite is weak or strong. These two structures yield an existential reading and a uniqueness reading ${ }^{11}$ respectively. The universal reading described in section 3 is missing.

Camelia has $\mathrm{a}_{\text {weak }}$ donkey/astrong donkey. She treats it well.

a. Weak reading: There is a donkey that Camelia owns and that she treats well.

b. Strong reading: Camelia has exactly one donkey and she treats it well.

The narrow pseudo-scope reading is not predicted either. PCDRT only predicts a wide pseudo-scope universal or existential reading. This omission from the system is intentional; indeed, in a discussion of number-neutral theories of donkey anaphora, Brasoveanu (2008) gives the example in (40), which at first blush suggests the absence of such readings.

11 Interestingly, the uniqueness reading is obtained as a combination of the strong indefinite and the singular feature on the anaphora. In principle thus, and assuming that plural features are semantically vacuous, such sentences as "*Camelia has $a_{\text {strong }}$ donkey and she treats them well" should be fine. 
Gaps in the interpretation of pronouns

(40) Every man who brought a friend to the party introduced him to every movie star there.

$\not \rightarrow$... is such that he introduced each movie star to a (possibly different) friend.

(40) does not seem to be compatible with any friend not being presented to every movie star, as the narrow pseudo-scope existential reading would have it. This seems to go against the evidence presented here and in Solomon 2012 for the existence of such readings.

Here is a speculation as to the origin of this discrepancy: for a narrow pseudoscope reading to be available in my system, the operator (here, every movie star) has to take scope above the pronoun at LF. Scoping is not free and is subject to constraints, such as Scope Economy (Fox 1995), which prevents a scoping operation from being vacuous. For Scope Economy to have detectable effects, the notion of semantically vacuous moves needs to be defined in a rather narrow way, so that indirect effects, like scope parallelism in ellipsis, may nonetheless be observed. Following this lead, it is conceivable that scoping over a pronoun as in (40) counts as a semantically vacuous move. As preliminary evidence for this view, consider the example I used to illustrate the narrow pseudo-scope readings, repeated below in (41). This example is structurally similar to that in (40), except that the pronoun is itself embedded in an indefinite ( a piece of). Introducing a semantically contentful quantifier around the pronoun seems enough to trigger scoping of each above it, thus generating the narrow pseudo-scope reading.

(41) Every man who received a chocolate bar $_{1}$ gave [a piece of it $_{1}$ ] to each of his kids.

Champollion et al. 2017. The trivalent treatment of vagueness in the present paper is adapted from an original idea of Champollion et al. (2017). The main difference between my approach and theirs concerns the origin of the vagueness. In their approach, it is the type-shifter that turns a static quantifier into a dynamic quantifier that introduces the vagueness; the pronoun does not participate in its creation.

The type-shifter they propose is constructed from the two procedures for creating dynamic quantifiers that I presented in section 2, repeated below in (43). In its type-shifted meaning, every is true just in case both the existential reading of every - as generated by the denotation in (43a) - and the universal reading of every - as generated by the denotation in (43b) - are true, false when both are false, \# otherwise. The italicized bits in the last sentence are the important ones. We already know the shortcomings of the denotations in (43): they only generate wide pseudo-scope readings. Consequently, so does the type shifter in (42). 
$\llbracket \uparrow$ every $\alpha \beta \rrbracket= \begin{cases}\text { true } & \text { if } \llbracket \text { every }_{\text {uni }} \alpha \beta \rrbracket=\text { true and } \llbracket \text { every } \alpha \beta \rrbracket=\text { true } \\ \text { false } & \text { if } \llbracket \text { every }_{\text {uni }} \alpha \beta \rrbracket=\text { false and } \llbracket \text { every } \alpha \beta \rrbracket=\text { false } \\ \# & \text { otherwise }\end{cases}$

a. $\llbracket$ every $_{\mathrm{ex}} \alpha \beta \rrbracket=\forall x \in \llbracket \alpha \rrbracket, \neg(\llbracket \alpha \rrbracket(x) \wedge \neg \llbracket \beta \rrbracket(x))$

b. $\llbracket$ every $_{\text {uni }} \alpha \beta \rrbracket=\forall x \in \llbracket \alpha \rrbracket, \llbracket \alpha \rrbracket(x) \wedge \llbracket \beta \rrbracket(x)$

To see this more clearly, consider once more the sentence in (41). Also consider a context where nine men received just one chocolate bar and gave a piece of that chocolate bar to all their kids. Finally, one man received two chocolate bars and used one chocolate bar for half his kids and the other for the other half. The sentence (41) is judged true in this context. By contrast, the type-shifter in (42) predicts it to be false: since the kids of the last man do not get a piece from all chocolate bars, the universal wide pseudo-scope reading in (43b) is false; since not all kids get a piece from the same chocolate bar, the existential narrow pseudo-scope reading in (43a) is false. In conclusion, the type-shifter proposed by Champollion et al. (2017) does not predict the narrow pseudo-scope readings, and this independently of the adoption of trivalence.

\section{Conclusion}

In the introduction, I presented three challenges posed by pronouns anteceded by indefinites: how referents are accessed by those pronouns, what truth-conditions they give rise to, and what pragmatic factors affect these truth-conditions. This paper contributed to the second challenge by presenting two readings that were not generated by previous accounts: universal cross-conjunction readings and narrow pseudo-scope readings. To account for these readings, I proposed that pronouns have vague reference. Supplemented with only standard rules of Alternative Semantics, the system was able to derive the missing readings.

While this account is sufficient to capture the facts presented here, it is incomplete insofar as it does not take a stance on the other two challenges. For instance, the analysis completely lacks a theory of the pragmatics of the ambiguity, i.e. the reading preferences observed by Kanazawa (1994) and their relative strength. In the view developed here, such preferences would have to be derived from the pragmatics of trivalence. How this can be done is a question left open to future research.

Another question left open here is the question of whether a wide pseudo-scope reading is ever available, when the operator is clearly scoping above the pronoun (cf discussion of (40)). The analysis here predicts this to be impossible. While I do not have unambiguous evidence of this, I suspect it is possible. If it is, adaptations to the current system of vagueness projection would need to be made. 
Gaps in the interpretation of pronouns

\section{References}

van den Berg, Martin. 1996. Dynamic generalized quantifiers. In Jaap van der Does \& Jan van Eijck (eds.), Quantifiers, logic and language, 63-94. Stanford University.

Brasoveanu, Adrian. 2007. Structured nominal and modal reference. New Jersey: Rutgers University PhD dissertation. doi:10.1017/CBO9781107415324.004.

Brasoveanu, Adrian. 2008. Donkey pluralities: Plural information states versus non-atomic individuals. Linguistics and Philosophy 31(2). 129-209. doi:10.1007/s10988-008-9035-0.

Brasoveanu, Adrian \& Donka Farkas. 2011. How indefinites choose their scope. Linguistics and Philosophy 34(1). 1-55. doi:10.1007/s10988-011-9092-7.

Champollion, Lucas, Dylan Bumford \& Robert Henderson. 2017. Donkeys under discussion. Semantics and Pragmatics (to appear).

Chierchia, Gennaro. 1995. Dynamics of meaning. University of Chicago Press. doi:10.7208/chicago/9780226104515.001.0001.

Cooper, Robin. 1979. The interpretation of pronouns. Syntax and Semantics 10. 61-92.

Foppolo, Francesca. 2008. The puzzle of donkey anaphora resolution. In Anisa Schardl, Martin Walkow \& Muhammad Abdurrahman (eds.), North east linguistics society (NELS), vol. 38, 297-310. GLSA.

Fox, Danny. 1995. Economy and scope. Natural Language Semantics 3(3). 283-341. doi:10.1007/BF01248820.

Geach, Peter Thomas. 1964. Reference and generality. Cornell University Press.

Geurts, Bart. 2002. Donkey business. Linguistics and Philosophy 25(2). 129-156.

Groenendijk, Jeroen \& Martin Stokhof. 1991. Dynamic predicate logic. Linguistics and Philosophy 14(1). 39-100. doi:10.1007/BF00628304.

Heim, Irene. 1982. The semantics of definite and indefinite noun phrases. Amherst, MA: University of Massachusetts, Amherst PhD dissertation.

Kamp, Hans \& Barbara Partee. 1995. Prototype theory and compositionality. Cognition 57(2). 129-191.

Kanazawa, Makoto. 1994. Weak vs. strong readings of donkey sentences and monotonicity inference in a dynamic setting. Linguistics and Philosophy 17(2). 109-158. doi:10.1007/BF00984775.

Kratzer, Angelika \& Junko Shimoyama. 2017. Indeterminate pronouns: The view from japanese. In Contrastiveness in information structure, alternatives and scalar implicatures, 123-143. Springer.

Križ, Manuel. 2016. Homogeneity, non-maximality, and all. Journal of Semantics 33(3). 493-539. doi:10.1093/jos/ffv006.

Muskens, Reinhard. 1996. Combining montague semantics and discourse represen- 
tation. Linguistics and Philosophy 19(2). 143-186. doi:10.1007/BF00635836.

Reinhart, Tanya. 1997. Quantifier scope: How labor is divided between qr and choice functions. Linguistics and Philosophy 20(4). 335-397.

Root, Rebecca Louise. 1986. The semantics of anaphora in discourse. Austin, Texas: University of Austin, Texas PhD dissertation.

Rooth, Mats. 1985. Association with focus. Amherst, Massachusetts: University of Massachusetts, Amherst PhD dissertation.

Schlenker, Philippe. 2012. The semantics/pragmatics interface. In Cambridge handbook of semantics, Cambridge: Cambridge University Press.

Solomon, Mike. 2012. Donkey readings and delayed quantification. New York: New York University MA thesis.

Keny Chatain

MIT Linguistics and Philosophy

32 Vassar Street 32-D806

MA02139 Cambridge

keny.chatain@gmail.com 